coronary heart disease. Among night workers, we observed no consistent exposure-response relations for any quantitative night work characteristic. However, men, but not women night workers showed an overall increased incidence rate ratio of coronary heart disease of 1.22 (95\% confidence interval 1.07-1.39) compared with dayworkers that could not be explained by medical or lifestyle factors.

Conclusion Our observation of no exposure-response relation between several quantitative night work characteristics and coronary heart disease does not provide support for an association between night work and coronary heart disease warranting specific night work schedules to prevent coronary heart disease.

\section{RF-187 NIGHT SHIFT WORK INCREASES THE RISK OF DIABETES: A 17-YEAR FOLLOW-UP COHORT STUDY AMONG ASIAN HEALTHCARE WORKERS}

${ }^{1}$ Wan-Chin Chen, Hsiao-Yu Yang. 'National Taiwan University College of Public Health, Taiwan

\subsection{6/OEM-2021-EPI.390}

Introduction In East Asian countries with highly developed economies, the prevalence of diabetes is rapidly increasing. Long working hours and shift work are suspected to be important risk factors in East Asia. A long-term follow-up study is warranted to clarify the relationship between diabetes, working hours, and shift work.

Objectives To evaluate the association of night shift work and risk of diabetes among Asian healthcare workers, and to explore their dose-response relationship using actual hours of night shift work monthly rather than cumulative years with night shift work.

Methods A retrospective cohort study was conducted among employees in a tertiary medical centre in central Taiwan. From 2002-2019, there were 7767 participants with a mean $( \pm S D)$ age of $27.9( \pm 7.0)$ years, and $89.6 \%$ of them were women. We collected information related to annual health check-ups, medical charts, and annual working hours, including during day, evening, and night shifts. We divided working hours into three categories (less than 60 hours per month, 60 to 100 hours per month, more than 100 hours per month). We estimated hazard ratios and 95\% confidence intervals (CIs) for incident diabetes using Cox proportional hazards models, adjusting for age, sex, and body mass index.

Results 309 (3.98\%) incident cases of diabetes occurred during 56,799 person-years at the 17-year follow-up. In the age- and sex-adjusted model, the HR (95\% CI) for those who worked night shifts for more than 100 hours per month was 2.062 (1.179 to 3.608) compared with those who worked fewer than 60 hours. In the stratified analysis, the association between night shift work and diabetes was significant among those aged $<40$ years, females, and obese participants.

Conclusions Among Asian healthcare workers, night shift work is associated with the incidence of diabetes in a dose-dependent manner. This finding could identify workers at high risk of diabetes to provide preventive strategies.

\section{Work organization and Return to Work}

\section{RF-37 ASSOCIATION BETWEEN MENTAL HEALTH SYMPTOMS AND SHIFTWORK AMONG FILIPINO WOMEN FACTORY WORKERS}

${ }^{1}$ Jinky Leilanie Lu, Sophia Francesca Lu. ${ }^{1}$ National Institutes of Health, University of the Philippines, Philippines

\subsection{6/OEM-2021-EPI.391}

Introduction This study aimed to look at the association between working at night and mental health symptoms, and mediating factors to this association.

Methods This study looked at a database of 500 factory workers, about $90 \%$ of which were females. It originally investigated hazard exposures and occupational health of workers in export processing zones in the Philippines. The database included variables relating to work schedule such as night shift, overtime, and extended work, as well as mental and psychological health indices through a survey questionnaire.

Results The study showed that work schedule, work load and occurrence of mental health symptoms are associated with night schedule. The crude odds ratio for the association between shift schedule and frequency of occurrence of mental health symptoms is $2.13(0.77-5.81)$. This means that without adjusting for confounders, those who work in the evening are 2.13 times more likely to have frequent occurrence of mental health symptoms as compared to those who work in the morning. Specifically, among females, those who work at night are 2.97 times more likely to have frequent occurrences of mental health symptoms compared to those who work in the morning. Those who are frequently exposed to occupational hazards are 5.78 (1.17-28.71) times more likely to have frequent mental health symptoms as compared to those who are not. The evidence for this association is strong.

Conclusion The study has shown that among Filipino women factory workers, nightshift work is associated with mental health symptoms.

\section{RF-182 ADOLESCENT TRAINEES WITH LEARNING DISABILITIES ON OCCUPATIONAL HEALTH AND SAFETY: PERCEPTION OF RISKS AND GENDER DIFFERENCES}

${ }^{1}$ Myriam Bérubé, Marie Laberge, Aurélie Tondoux. 'University of Montreal, Canada

\subsection{6/OEM-2021-EPI.392}

Introduction Adolescents with learning disabilities are vulnerable in terms of occupational health and safety (OHS). The Quebec Work-Oriented Training Path (WOTP) prepares them for the job market by offering pre-employment internships. Those are often typically feminine or masculine, and attract students according to their gender stereotypes. This segregation implies different OHS risks for women or men, who have chosen different environments. Considering this complex context, a research team is developing digital tools to improve the OHS management in the WOTP.

Objectives The first step is to identify what students currently understand about OHS, the risks present in their internships, and how gender influence these elements. 
Methods A short answer questionnaire addressing these topics was completed by 131 WOTP students $(38$ girls and 93 boys).

Results The three most popular work environments were garages (tire installer, 17 boys), retail stores (stock handler, 21 boys, 9 girls) and restaurants (fast-food counter attendant and cook's helper, 17 boys, 6 girls). More than $40 \%$ of the girls are in typically female occupations: hair salon assistant (7), assistant childcare educator (5), grooming assistant (3) and recreation assistant (1). When asked if they perceived risks in their internships, $50 \%$ of girls and $80 \%$ of boys say yes. All girls say they consider OHS important or very important, while $13 \%$ of boys are indifferent or consider it not very important. Concerning the OHS training received at the workplace, $38 \%$ of the girls and $50 \%$ of the boys stated that they had received some.

Conclusion These preliminary results suggest that girls and boys are not exposed to the same environments nor the same risks. The situation seems inequitable in terms of prevention and suboptimal for all, since a large proportion reported not having an OHS training. These shortcomings should be considered in the development of new tools.

\section{RF-322 RATES OF RETURN TO WORK AND WORK ABILITY FOLLOWING KNEE REPLACEMENT}

${ }^{1}$ Elena Zaballa, Georgia Ntani, E Clare Harris, Nigel K Arden, Cyrus Cooper, Karen WalkerBone. ${ }^{1}$ University of Southampton, United Kingdom

\subsection{6/OEM-2021-EPI.393}

Introduction There is limited evidence on the ability to sustain employment following knee replacement.

Objective To describe occupational status before and after unicompartmental (UKR) and total knee replacement (TKR) and to explore work ability after both types of knee arthroplasty.

Methods This study was set within the longitudinal Clinical Outcomes in Arthroplasty study. Eligible participants were those who were aged 18-64 years at the time of their primary arthroplasty and had at least 5 years' duration followup since. All eligible participants were mailed a questionnaire which enquired about: pre- and post-operative occupations and occupational exposures; time to reach best function; and leisure-time physical activities performed postoperation. We used Cox regression to estimate crude and adjusted hazard ratios with $95 \%$ confidence intervals to explore the role of work factors in determining post-operative work ability (CI).

Results 251 useable responses were received (61\% response) amongst whom 158 (63\%) returned to any work postoperatively. Most of these, 146 (92\%) had been working pre-operatively. Detailed occupational exposure information was available for 145 (94 UKRs, 51 TKRs) of the 158 who RTW. The mean follow-up post-operation was 6.4 years $(\mathrm{SD} \pm 1)$. Adjusted models showed that workers were at increased risk of leaving their job post-operation if an average working day involved: lifting/carrying $\geq 10 \mathrm{~kg}$ (HR: 4.57 95\%CI $1.52,13.79$ ) or climbing 30 flights of stairs/day (HR:3.52 95\%CI 1.29,9.59), as compared with workers not doing these activities. Mutually adjusted models showed that it was the lifting/carrying weights in excess of $10 \mathrm{~kg}$ that was most important (HR:3.6 95\% CI 1.1,11.3).
Conclusion Many people effectively return to work post kneereplacement. However, job retention is poorer in occupations that entail lifting weights and climbing flights of stairs. Subject to replication, these findings could imply a particular role for post-UKR/TKR rehabilitation amongst people needing to RTW in physically-demanding jobs.

\section{RF-326 DOES PARTICIPATION IN MODIFIED RETURN TO WORK DIFFER FOR IMMIGRANT COMPARED TO CANADIAN- BORN WORKERS IN BRITISH COLUMBIA, CANADA?}

Sonja Senthanar, 'Mieke Koehoorn, Lillian Tamburic, Stephanie Premji, Ute Bültmann, Chris McLeod. 'University of British Columbia, Canada

\subsection{6/OEM-2021-EPI.394}

Introduction The longer workers are off work, the less likely they are to successfully return to work. Modified return-towork (MRTW) provides earlier work reintegration after an injury with the goal of full recovery and shorter work disability durations.

Objective This study examined differences in MRTW participation for immigrant compared to Canadian-born workers with a work-related injury in British Columbia.

Methods Workers' compensation claims data linked with immigration records were used to identify immigrant (economic, family or refugees/other classifications) and Canadian-born workers with an accepted short-term disability claim between 2009-2015 for acute (fracture and concussion) and chronic injuries (back strain and connective tissue). Injury cohorts were restricted to 30 days on claim for a common disability window to measure MRTW participation. The outcome of MRTW was measured as never offered MRTW or an offer of MRTW within the first 30 days on benefits. Individuals with an offer beyond 30 days were excluded.

Results In adjusted logistic regression models, immigrant classification was associated with a decreased odds ratio (OR) of receiving an offer of MRTW within 30 days across injury cohorts. For example, for workers with back strain injuries: $\mathrm{OR}=0.97$ [95\%CI $0.88,1.07]$ for economic immigrants, $\mathrm{OR}=0.72[95 \% \mathrm{CI} 0.66,0.79]$ for family class immigrants, and $\mathrm{OR}=0.74[95 \% \mathrm{CI} 0.64,0.86]$ for refugee/other classifications. Similarly, for workers with a fracture injury: $\mathrm{OR}=0.94 \quad[95 \% \mathrm{CI} \quad 0.72,1.22]$ for economic immigrants, $\mathrm{OR}=0.84 \quad[95 \% \mathrm{CI} 0.64,1.11]$ for family class immigrants, and $\mathrm{OR}=0.42 \quad[95 \% \mathrm{CI} \quad 0.24,0.76]$ for refugee/other classifications.

Conclusion Understanding why immigrant workers are less likely to be offered MRTW compared to Canadian-born workers for the same injuries is worthy of further investigation, and in particular for workers who arrived to Canada as refugees. A focus on increasing earlier provision of MRTW for longer term claims has the potential to reduce work disability duration among immigrant workers.

\section{RF-298 OCCUPATIONAL RISK OF SALMONELLOSIS AND CAMPYLOBACTERIOSIS: A NATIONWIDE POPULATION- BASED REGISTRY STUDY}

${ }^{1}$ Janneke Duijster, Lapo Mughini-Gras, Eelco Franz, Jacques Neefjes. ${ }^{1}$ National Institute for Public Health and the Environment (RIVM), Netherlands

10.1136/OEM-2021-EPI.395 